\title{
Nursing students' perception of safety in clinical settings: From the quality and safety officer
}

\author{
Elizabeth E. Cooper * \\ School of Nursing and Health Professions, University of San Francisco, San Francisco, CA, United States
}

Received: March 3, 2017

Accepted: May 18, 2017

Online Published: May 24, 2017

DOI: $10.5430 /$ jnep.v7n10p91

URL: https://doi.org/10.5430/jnep.v7n10p91

\begin{abstract}
Teaching methods to improve the safety of care for patients has been a priority for nurse educators. This article discusses the student nurses' use of error reporting tools in the clinical setting, revealing study results completed by the Quality and Safety Officer in a School of Nursing and Health Professions. The aim was to report on the use of safety tools and the perception of safety issues in clinical settings identified by 121 prelicensure baccalaureate nursing students. Responses suggest that it is challenging for nursing students to report errors and near miss events. Barriers exist for the nursing student. The survey reveals difficulty in reporting but discloses that safety for the patient continues to be a primary concern for the nursing student.
\end{abstract}

Key Words: Quality and safety officer, Error safety reporting, Near miss reporting, Quality and safety education for nurses, Nurse educator

\section{INTRODUCTION}

Nurse educators work to provide educational experiences for nursing students that enhance opportunities focusing on increasing safety for patients. ${ }^{[1]}$ Over one decade ago, the Institute of Medicine (IOM) published, "To Err is Human" and issued in a new awareness of patient safety issues. Their groundbreaking reports, raised awareness of the need to increase quality and safety for all involved in healthcare. ${ }^{[2,3]}$ Health professionals feel the urgency to make changes that address this need in multiple settings. ${ }^{[1]}$ Baccalaureate nursing education is expected to continue to provide a high quality education, with the goal of ensuring that the new nurse graduate will be able to provide safe patient care in a variety of settings. ${ }^{[4]}$

Quality and Safety Education for Nurses (QSEN) is an example of an initiative developed to address the safety issues identified by the IOM. This Robert Wood Johnson Foundation funded initiative developed competencies to enhance prelicensure nursing education. The competencies include: safety, informatics, evidence-based practice, teamwork and collaboration, patient-centered care, and quality improvement. The goal of the QSEN framework is to provide clear definitions and competencies fostering safe patient care. These clear definitions and competencies provided nursing educators with a guide to weave QSEN throughout their nursing curriculum. The impact of weaving QSEN into the nursing curriculum was to transform student nurses' education to include integration of quality and safety, which in turn, will increase the quality of their contributions in healthcare. ${ }^{[5]}$

The school of nursing and health professions established a Quality and Safety Officer (QSO) role. This formal role was created as a response to the IOM and QSEN initiatives. The QSO role works to enhance safety and transparency in prelicensure nursing education by establishing an error and

\footnotetext{
* Correspondence: Elizabeth E. Cooper; Email: eecooper@usfca.edu; Address: School of Nursing \& Health Professions, University of San Francisco, 2130 Fulton Street San Francisco, CA 94117-1080, United States.
} 
near miss reporting system. ${ }^{[6]}$ In nursing practice, error and near miss safety tools provide a formal method of reporting. This reporting system is used in addition to the health care institution's safety reporting system. QSEN defines an error as either the execution of an act not proceeded as intended or as a planned event not completed correctly. A near miss is an event that is similar to an error but is discovered and never reaches the patient. ${ }^{[7]}$ The error and near miss reporting tools provide a method for the student to report these issues, which allows for a consistent method of error tracking for both the student and nurse educator.

The standard policy states that the nursing student and/or faculty report safety issues to the QSO. This reporting system is a strategy that leads to transparency in the school of nursing. Each semester, faculty and nursing students receive summaries of safety reports based on the reporting tool information. This introduces the concepts of transparency, blame-free reporting, and safety culture to the student and faculty members. ${ }^{[8]}$ Open feedback about reporting errors is important to the healthcare provider including the nursing student. This transparent safety culture leads to an increase in reporting and may increase patient safety. ${ }^{[9]}$

The QSO continuously monitors and evaluates the safety reporting system. The QSO obtains information regarding the knowledge, use, and availability of the reporting tools. In addition, the QSO monitors the student's perception of safety issues and communication in the clinical setting.

\section{METhOD}

To explore the incidence and the nursing students' perception of safety events and reporting, an exploratory online survey was conducted with 121 prelicensure nursing students in a 4-year baccalaureate program at a private university. This online survey was initially sent via e-mail to 464 baccalaureate- nursing students, with one additional reminder e-mail sent. A total of 121 students responded, representing a convenience response sample of a $26 \%$ rate. The survey was completed August 2016, before the fall semester began. The students based their responses on their clinical experience of spring 2016. Participation did not have any relationship to any course offered at the school of nursing. The university's institutional review board for protection of human subjects granted an exempt status to the exploratory survey.

\section{RESULTS}

Error and near miss information data were obtained along with perception of safety in clinical settings. Two 5-point Likert scales were employed to obtain two sets of information. One scale on the survey focused on the work area using strongly agree to strongly disagree rating system. The second scale of the survey focused on communication using always to never rating system.

\subsection{Demographics}

Students represented the nursing student at levels of sophomore (43\%), junior (38\%), and senior one level (19\%). Multiple clinical teaching site settings were represented. Examples of student clinical sites included: hospital sites, out patient clinics, community and mental health sites, and elementary, middle, and high school sites. The hospital setting was the largest represented at $88 \%$ (see Figure 1). Eighty-eight percent of the students reported that they were introduced to the nursing school's safety tools. This introduction to the nursing school's safety tools and policy took place in multiple settings, which included: clinical, theory course, online student school site, e-mail, and conversation with peers. Clinical and theory courses were the primary setting for students reported in this survey study at $69 \%$ and $59 \%$, respectively.

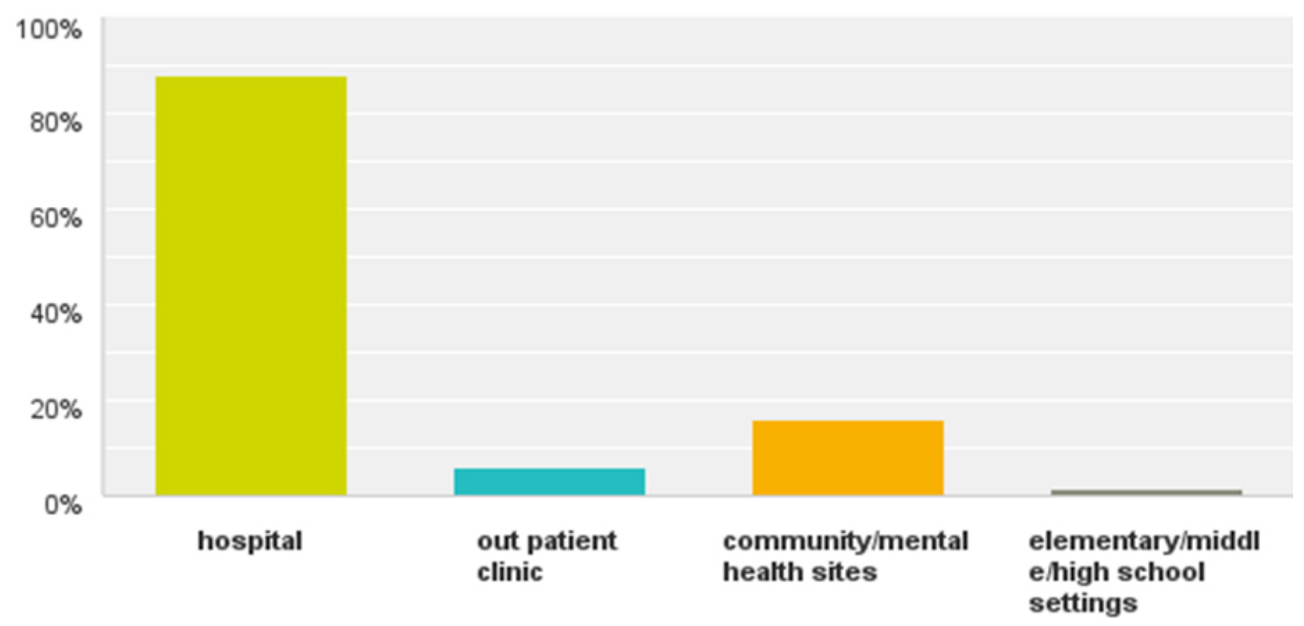

Figure 1. Types of clinical settings 
One question in the study (section 4, item 3) asked, did your clinical instructor discuss patient safety including the use of reporting tools? The majority of students (62\%) reported that their clinical instructor did discuss patient safety. These conversations mainly occurred in the post clinical conference session and on the clinical orientation day. $10 \%$ of students report that they did not talk about safety in the clinical setting and $27 \%$ report that they cannot recall.

\subsection{Safety feedback}

\subsubsection{Error reporting}

Another question in the survey asked, what types of errors did you encounter? The majority ( $82 \%$ ) of the students reported they had never encountered an error. But for the students that had been exposed to an error, medication administration was the most common type. The other types of errors identified were: wrong treatment provided to the patient, omission of treatment, and needle stick (see Figure 2).

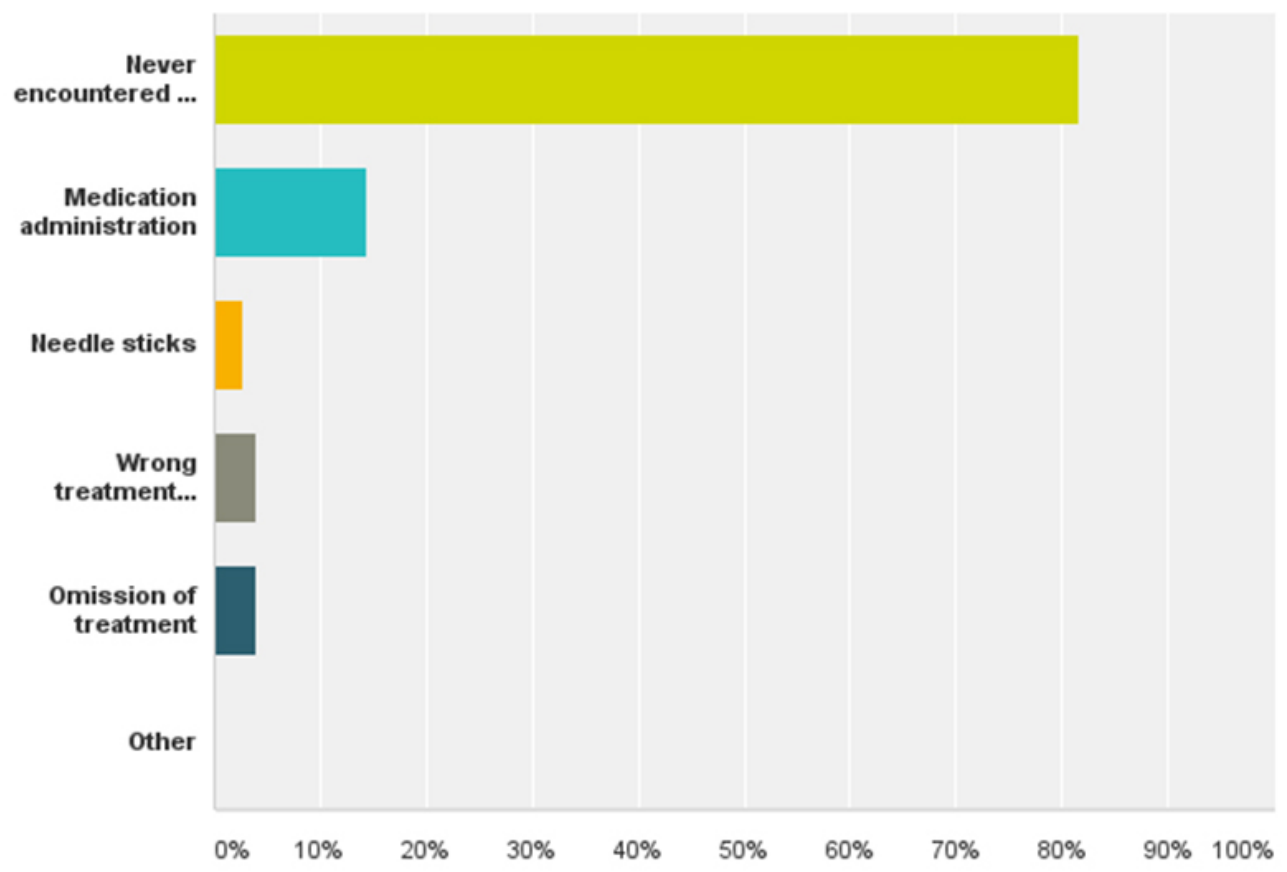

Figure 2. Types of errors

The error safety tool was developed to provide a consistent method of reporting for the nursing students. The safety tool was available as an online document or paper version for the nursing student. The one page document provided a template for the nursing student to complete to summarize the error or near miss event. A question in the survey asked, for those nursing students that an error was encountered, was a safety tool completed? For this question, $21 \%$ of the students that reported encountering an error, but $17 \%$ of the $21 \%$ of nursing students, did not complete the safety-reporting tool. Multiple reasons were provided for not following the school's safety protocol. Reasons included: no harm to patient, so no need to report; was not the student's error; nurse that was working with student encouraged student not to report; and fear of outcome for the student.

The students in the study reported that the reporting tool was easy to access and easy to use. The majority of students responding to using the tool reported that returning the com- pleted tool to the QSO was easy. Methods such as e-mail, office mail, or delivery under office door were reported.

\subsubsection{Near-miss reporting}

A near miss event is an event that does not actually occur or touch the patient. An example could include almost giving the patient the wrong dose of a medication, but realizing the mistake before you actually give the medication. Almost an error, but the healthcare provider corrects the error before it reaches the patient. The majority (86\%) of nursing students in this survey, report that they never encountered a near miss event. Of those that did, a near miss or almost medication error event led the list (see Figure 3). Almost providing: the wrong treatment, wrong patient, wrong times were also mentioned. The near-miss reporting tool was reported as easy to use, easy to give to the QSO, but rarely completed.

\subsection{Work area perception}

Safety perception, as reported by the nursing students on the clinical unit, was examined using a 5-point Likert scale (see 
Table 1). The students reported that the clinical nurse and student were actively doing things to improve patient care and that safety for the patient is a common topic of discussion. The majority of students, $90 \%$, reveal that safety is their focus during a clinical shift. However, $12 \%$ of nursing students reported that patient safety is sometimes sacrificed in order to get the work completed. The participants reported that the patient also had safety as their main goal too.

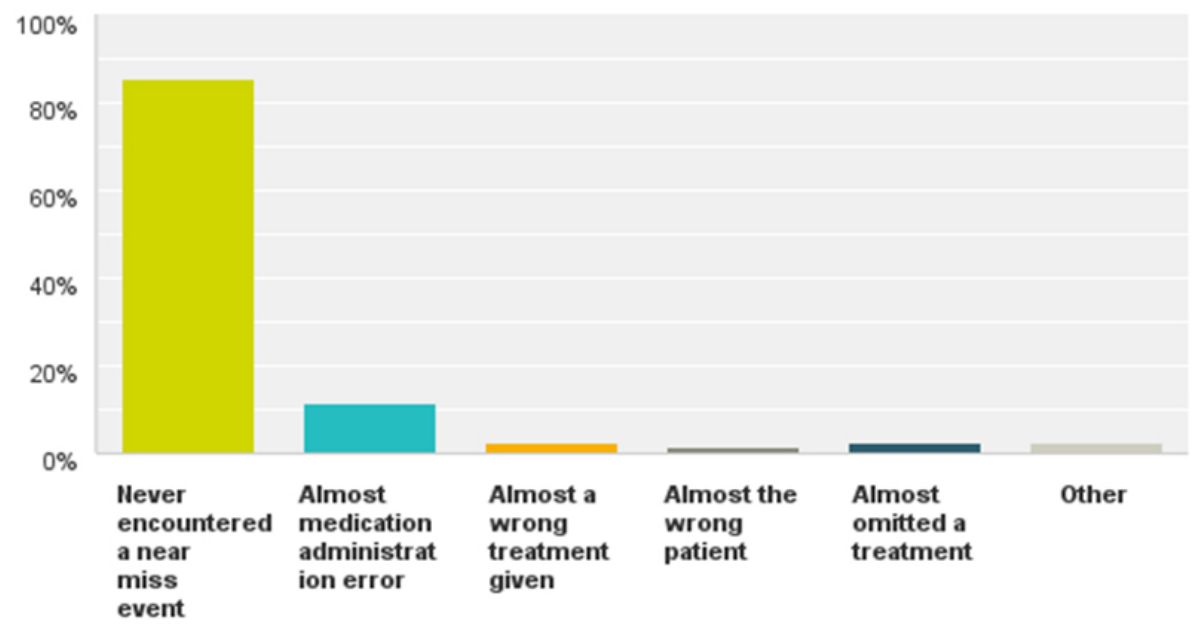

Figure 3. Near miss events

Table 1. Work area safety perception

\begin{tabular}{|c|c|c|c|c|}
\hline Safety & $\begin{array}{l}\text { Strongly agree } \\
\text { or agree } \\
(\%)\end{array}$ & $\begin{array}{l}\text { Neither agree } \\
\text { or disagree } \\
(\%)\end{array}$ & $\begin{array}{l}\text { Disagree or } \\
\text { strongly } \\
\text { disagree (\%) }\end{array}$ & $\mathbf{M}^{*}(\mathbf{S D})$ \\
\hline \multicolumn{5}{|l|}{ Your work area perception... } \\
\hline Nurses \& students are actively doing things to improve patient safety & 89 & 10 & 1 & $4.15(0.63)$ \\
\hline The nurses \& students discuss safety issues & 83 & 13 & 5 & $4.08(0.79)$ \\
\hline As a student, I have a safety focus for my shift & 100 & 0 & 0 & $4.61(0.49)$ \\
\hline My patient has a safety focus for my shift & 78 & 10 & 0 & $4.26(0.63)$ \\
\hline Patient safety is never sacrificed to get more work done & 78 & 9 & 12 & $4.05(1.03)$ \\
\hline My clinical instructor focuses on safety issues & 96 & 3 & 1 & $4.39(0.60)$ \\
\hline There are safety problems on this unit & 34 & 28 & 39 & $2.91(0.99)$ \\
\hline Students feel that mistakes are held against them & 43 & 23 & 34 & $3.06(1.07)$ \\
\hline Nurses feel that mistakes are held against them & 41 & 28 & 31 & $3.09(0.99)$ \\
\hline Our policies and procedures are good at preventing errors from happening & 90 & 10 & 0 & $4.05(0.50)$ \\
\hline I feel that I am providing safe care & 99 & 1 & 0 & $4.31(0.49)$ \\
\hline
\end{tabular}

*The items were coded as strongly disagree (1), disagree (2), neither (3), agree (4), and strongly agree (5).

The clinical instructor focused on safety issues the majority of the time. Students report that there are safety issues on the clinical unit at $43 \%$. From the students' perception, both the clinical nurse and student feel that errors are held against them.

\section{COMMUNiCATion \& SAFETY REPORTING PERCEPTION}

Communication and safety reporting perception on the clinical unit was examined using a 5-point Likert scale (see Table
2). Feedback from the nursing student reveals that the student perceives that the clinical nurse and student discuss multiple ways to prevent errors for their patient. Forty-nine percent of nursing students report that they feel free to speak up to their nurse or clinical instructor, if they see something that may negatively effect the patient. The students' reported that the clinical nurses also feel they can speak freely in the healthcare environment. The majority of students are aware of an error made by themselves, other students, or by the clinical nurses. They report that the majority of the time 
an incident report addressing the error is completed in the clinical setting and in addition, the safety-reporting tool is completed too. Fifty percent of the participants reported that near miss events are reported.

Table 2. Communication \& safety reporting perception

\begin{tabular}{|c|c|c|c|c|}
\hline Safety & $\begin{array}{l}\text { Always or most } \\
\text { of the time }(\%)\end{array}$ & $\begin{array}{l}\text { Sometimes } \\
(\%)\end{array}$ & $\begin{array}{l}\text { Rarely or } \\
\text { never }(\%)\end{array}$ & $\mathbf{M}^{*}(\mathrm{SD})$ \\
\hline \multicolumn{5}{|l|}{ Communication and safety reporting... } \\
\hline $\begin{array}{l}\text { Students and nurses on this unit, discuss ways to prevent errors } \\
\text { from happening }\end{array}$ & 60 & 34 & 6 & $3.72(0.84)$ \\
\hline $\begin{array}{l}\text { Students feel free to speak up if they see something that may } \\
\text { negatively affect the patient }\end{array}$ & 49 & 39 & 11 & $3.49(0.84)$ \\
\hline $\begin{array}{l}\text { Nurses appear free to speak up if they see something that may } \\
\text { negatively affect the patient }\end{array}$ & 73 & 19 & 8 & $3.99(0.91)$ \\
\hline I am aware of errors made by myself, other students or nurses & 74 & 16 & 10 & $3.77(0.94)$ \\
\hline $\begin{array}{l}\text { Students are afraid to ask questions if something does not seem } \\
\text { right }\end{array}$ & 24 & 46 & 29 & $2.91(0.89)$ \\
\hline $\begin{array}{l}\text { Students are informed about errors that happened during their } \\
\text { semester }\end{array}$ & 38 & 28 & 33 & $3.08(1.19)$ \\
\hline When an error is made, a hospital incident report is completed & 77 & 17 & 5 & $4.01(0.82)$ \\
\hline $\begin{array}{l}\text { When an error is made, a nursing school specific error report is } \\
\text { completed }\end{array}$ & 80 & 9 & 11 & $4.01(0.91)$ \\
\hline Near miss events are reported & 55 & 34 & 11 & $3.58(1.06)$ \\
\hline $\begin{array}{l}\text { When an error is made but has no potential harm to the patient, how } \\
\text { often do students report it }\end{array}$ & 33 & 44 & 22 & $3.19(0.92)$ \\
\hline $\begin{array}{l}\text { When an error is made but could harm the patient but does not, how } \\
\text { often do students report it }\end{array}$ & 55 & 34 & 11 & $3.61(0.87)$ \\
\hline Students are concerned about errors & 97 & 3 & 0 & $4.66(0.52)$ \\
\hline Nurses are concerned about errors & 91 & 9 & 0 & $4.43(0.65)$ \\
\hline Clinical instructors support students when errors are committed & 79 & 18 & 3 & $4.11(0.79)$ \\
\hline
\end{tabular}

*The items were coded as never(1), rarely (2), sometimes(3), most of the time(4), and always(5).

The nursing students in the survey report that error reports are completed more often if harm to the patient has actually been perceived. Also reported that the clinical nurse and student are concerned with errors the majority of the time. The clinical instructor is supportive to the student when an error occurs, most of the time (45\%) and always (34\%).

\section{DiscuSsion}

The goal of this study is to obtain perception of safety reporting information from the baccalaureate-nursing students regarding their use in the clinical setting. The QSO's aim was to increase transparency within the school of nursing and health professions and to measure the effectiveness of the safety tool.

The majority of participants $(88 \%)$ report that they were oriented to the reporting tools. The error reporting tools are located in myriad locations to enhance availability. However, the use of tools is not consistent when an error occurs in the clinical setting. The perception of reporting an error through the school's error reporting system is high at $81 \%$. Of the $21 \%$ of students that reported that they encountered an error, $17 \%$ did not report the error. The perception does not match the actual action. Nursing students that acquire the knowledge of what to report when an error or near miss occurs and then how to report the error actually can create a safe environment for themselves and their patients. ${ }^{[7]}$

The survey demonstrated that communication can be improved. While the nursing student participants report that they discuss errors with their clinical nurse, they also reveal that many do not feel free to speak up if something may negatively affect the patient. The clinical instructor, as a leader, needs to encourage communication among the student, clinical nurse, and themselves. Research has documented that leadership support, for the health care providers, including the nursing students, can positively affect safe patient care issues. ${ }^{[10]}$ The clinical instructor can help to bridge the gap between the nursing student and clinical nurse, in order to increase communication. 
This study reveals that medication errors are the most common category in both error and near-miss events for our nursing students that actually experience an error or near miss event. For the error report, $26 \%$ of the nursing students reported that they encountered an error. Medication error was identified $15 \%$ of the time. For the near miss report, $22 \%$ of nursing students reported they encountered a near miss event with medication near miss occurring $12 \%$ of the time. These results mirror what is happening in the health care arena for the clinical nurse. Medication errors represent the majority of preventable errors and continue to be a major concern for our patients' safety. ${ }^{[11]}$ Increasing discussions about medication administration should be a priority. The clinical instructor needs the support of the didactic/theory instructors to continue to focus on safety. Some examples include: stressing medication calculations, demonstrating methods of administration, and encouraging discussions among nursing students regarding their experiences administrating medications in the clinical setting.

Barriers students reveal for reporting errors are consistent with barriers that nurses and physicians report. ${ }^{[10]}$ Fear of failing, blame, embarrassment, and guilt are all identified in this study. Another issue that affects only the student is the occasional pressure of not reporting errors from the clinical nurse, as reported in this study. The nursing student had conflicting messages: one from the clinical nurse not to report errors and one from the clinical instructor to report errors. These conflicting messages from two people in power serve to confuse the nursing student.

The QSO in the school of nursing and health professions has worked on the safety-reporting project with the goal of increasing transparency and creating an error reporting method. The use of the reporting system continues to be a challenge to the nursing students. Support from leadership, including faculty and clinical instructors, needs to be increased. Communication methods need to be strengthened between the nursing student, clinical nurse, and clinical instructor. A similar study was conducted in 2013 and the results are consistent with this current study. ${ }^{[12]}$

\section{Limitations}

This study revealed that there are limitations in regards to this exploratory survey. The main limitation issue is that the student level group of senior two, capstone students, are not represented. Capstone students characterize the student in their final clinical rotation. Second, a small sample size in the study of 121 with the response rate of $26 \%$. Another limitation identified was that not all participants responded to every question.

\section{Conclusion}

Patient safety needs to be our primary goal for all who work in healthcare. As nurse educators, arming our students with tools to keep safety as a focus in their practice may increase safety for our patients. Providing the error and near miss reporting tools is one example for our nursing students. Transparency and safety issues must be introduced at the beginning of our students' educational experience and then carried through to the end. Fostering a blame-free, safe environment for our nursing students encourages transparency at school and in the clinical setting.

The goal of safety reporting and increasing transparency can only be met if the buy-in is high at both the faculty and student level. Buy-in means that the participants, in this case the faculty and nursing students, believe in the goal and agree to work towards achieving the goal. Communication with the faculty and nursing students is essential for buy-in.

Throughout the last decade, healthcare providers have worked to answer the IOM's call for increasing safety for our patients. Nurse educators have answered the call by instituting the QSEN initiative in their nursing educational programs. Creating and implementing an error and near miss reporting protocol is one example of increasing transparency and safety in a school of nursing and health professions. In spite of the effort of the QSO and the nurse educators, this survey demonstrates there is room for improvement in error reporting. In order to have transparency of errors in the healthcare setting, the introduction and implementation must begin in nursing school. The more the nursing educators talk about errors, the more accepted the communication would become to nursing students. Supporting the nursing student when an error occurs should be our emphasis in order for transparency to occur at the patient level. Nurse educators and nursing students must critically evaluate the amount of time spent on transparency of the clinical experience.

\section{CONFlicts of InTERest Disclosure}

The author declares that there is no conflict of interest.

\section{REFERENCES}

[1] Barnsteiner J. Safety. In G. Sherwood \& J. Barnsteiner (Eds.) Quality and Safety in Nursing: A Competency Approach to Improving Outcomes. West Sussex, UK: Wiley-Blackwell. 2012. 149-169 p.
[2] Institute of Medicine. To err is human: Building a safer health system. Washington, DC: National Press. 1999.

[3] Institute of Medicine. Health professions education: A bridge to quality. Washington, DC: National Academic Press; 2003. 
[4] American Association of Colleges of Nursing. The essentials of baccalaureate education for professional nursing practice. Washington, DC. 2008.

[5] Cronenwett L, Sherwood G, Barnsteiner J, et al. Quality and safety education for nurses. Nursing Outlook. 2007; 55(3): 122131. PMid:17524799 http://dx. doi .org/10.1016/j. outlook .2007 .02 .006

[6] Cooper E. Establishing the role of a quality and safety officer in a school of nursing. In L. Caputi (Ed). Building the Future of Nursing. Baltimore, MD: Wolters Kluwer Lippincott Williams \& Wilkins. 2014. 45-50 p.

[7] Sherwood G. Driving forces for quality and safety: Changing mindsets to improve health care. In G. Sherwood \& J. Barnsteiner (Eds.) Quality and Safety in Nursing: A Competency Approach to Improving Outcomes. West Sussex, UK: Wiley-Blackwell. 2012. 3-21 p.

[8] Cooper E. A spotlight on strategies for increasing safety reporting in nursing education. The Journal of Continuing Education in Nursing. 2012; 43(4): 162-168. PMid:22148176 https://doi.org/10.3 928/00220124-20111201-02
[9] Vrbnjak D, Denieffe S, O'Gorman C, et al. Barriers to reporting medication errors and near misses among nurses: A systematic review. International Journal of Nursing Studies. 2016; 63: 162-178. PMid:27637011 https://doi.org/10.1016/j.ijnurstu. 201 6.08 .019

[10] Castel E, Ginsburg L, Zaheer S, et al. Understanding nurses' and physicians' fear of repercussions for reporting errors: Clinician characteristics, organization demographics, or leadership factors? BMC Health Services Research. 2015; 15(326).

[11] Muroi M, Shen J, Angosta A. Association of medication errors with drug classifications, clinical units, and consequence of errors: Are they related? Applied Nursing Research. 2017; 33: 180-185. PMid:28096015 https://doi.org/10.1016/j .apnr.2016.12 .002

[12] Cooper E. From the school of nursing quality and safety officer: Nursing students' use of safety reporting tools and their perception of safety issues in clinical settings. Journal of Professional Nursing. 2013; 29(2): 109-116. https://doi.org/10.1016/j.profnurs .2012 .12 .005 